\title{
Efficient Generation of Singlet Oxygen and Photooxidation of Sulfide into Sulfoxide via Tuning the Ancillary of Bicyclometalated Iridium(III) Complexes
}

\author{
Li-Ping Li, and Bao-Hui Ye* \\ ${ }^{\dagger} \mathrm{MOE}$ Key Laboratory of Bioinorganic and Synthetic Chemistry, School of Chemistry, Sun Yat-sen \\ University, Guangzhou 510275, China.
}

1. Synthesis of $3 g$. Phenylboronic acid $(0.6 \mathrm{mmol})$ was added to a solution of DMF $(2 \mathrm{~mL})$ containing 4-methylbenzenethiol $(0.5 \mathrm{mmol})$ in a Schlenk tube at room temperature under oxygen condition. Subsequently, $\mathrm{Cs}_{2} \mathrm{CO}_{3}(1 \mathrm{mmol})$ and $\mathrm{CuI}(0.05 \mathrm{mmol})$ were added. The mixture was heated under $90{ }^{\circ} \mathrm{C}$ for $12 \mathrm{~h}$ and then cooled to room temperature. Water $(10 \mathrm{~mL})$ was added to the reaction mixture and EtOAc $(3 \times 10 \mathrm{~mL})$ were used to extract the crude product. After removing the solvent, the resulting crude was purified by column chromatography with petroleum ether/ethyl acetate $(20: 1)$ as eluent to give as a yellow oil 3g. Yield, $74 \mathrm{mg}, 69 \%$. Anal. calcd for $\mathrm{C}_{13} \mathrm{H}_{12} \mathrm{OS}$ : C 72.19, H 5.59, S 14.82; found: C 72.23; H 5.61, S 14.81\%. ESI-MS: $m / z=215.06[\mathrm{M}-\mathrm{H}]^{+} .{ }^{1} \mathrm{H}$ NMR $\left(400 \mathrm{MHz}, \mathrm{CDCl}_{3}\right): \delta 7.41(\mathrm{~d}, J=$ $7.9 \mathrm{~Hz}, 1 \mathrm{H}), 7.26-7.20(\mathrm{~m}, 2 \mathrm{H}), 7.17-7.11(\mathrm{~m}, 1 \mathrm{H}), 7.09-7.05(\mathrm{~m}, 2 \mathrm{H}), 6.91(\mathrm{~d}, J=0.9 \mathrm{~Hz}, 1 \mathrm{H})$, 6.81-6.77 (m, 1H), 6.45 (s, 1H), 2.37 (s, 3H). $\left.{ }^{13} \mathrm{C} \mathrm{NMR} \mathrm{(101} \mathrm{MHz,} \mathrm{CDCl}_{3}\right): \delta 157.25,143.19,136.79$, $136.48,129.25,126.66,126.66,126.60,126.60,122.43,116.48,112.84,21.64$.

2 Photooxidation of Sulfide into Sulfoxide.

For 4a. Yield, $69 \mathrm{mg}, 98 \%$. ESI-MS: $m / z=141.03[\mathrm{M}+\mathrm{H}]^{+} .{ }^{1} \mathrm{H} \mathrm{NMR}\left(400 \mathrm{MHz}, \mathrm{CDCl}_{3}\right): \delta 7.57(\mathrm{t}, J=$ $7.5 \mathrm{~Hz}, 2 \mathrm{H}), 7.49-7.39$ (m, 3H), 2.65 (s, 3H). ${ }^{13} \mathrm{C} \mathrm{NMR}\left(101 \mathrm{MHz}, \mathrm{CDCl}_{3}\right): \delta 145.53,131.04,129.41$, $129.38,123.67,123.49,43.7$.

For 4b. Yield, $83 \mathrm{mg}, 98 \%$. ESI-MS: $m / z=171.04[\mathrm{M}+\mathrm{H}]^{+} .{ }^{1} \mathrm{H}$ NMR $\left(400 \mathrm{MHz}, \mathrm{CDCl}_{3}\right): \delta$ 7.57-7.62 $(\mathrm{m}, 2 \mathrm{H}), 7.01-7.06(\mathrm{~m}, 2 \mathrm{H}), 3.86(\mathrm{~s}, 3 \mathrm{H}), 2.70(\mathrm{~s}, 3 \mathrm{H}) .{ }^{13} \mathrm{C} \mathrm{NMR}\left(101 \mathrm{MHz}, \mathrm{CDCl}_{3}\right): \delta 161.95$, $136.54,125.44,125.42,114.85,114.83,55.58,44.04$.

For 4c. Yield, $83 \mathrm{mg}, 98 \%$. ESI-MS: $m / z=171.04[\mathrm{M}+\mathrm{H}]^{+} .{ }^{1} \mathrm{H}$ NMR $\left(400 \mathrm{MHz}, \mathrm{CDCl}_{3}\right): \delta 7.79(\mathrm{~d}, J=$ 7.7 Hz, $1 \mathrm{H}), 7.47-7.38$ (m, $1 \mathrm{H}), 7.20-7.12(\mathrm{~m}, 1 \mathrm{H}), 6.90$ (d, J=8.2 Hz, $1 \mathrm{H}), 3.86(\mathrm{~s}, 3 \mathrm{H}), 2.75$ (s, 3 H). ${ }^{13} \mathrm{C} \mathrm{NMR}\left(101 \mathrm{MHz}, \mathrm{CDCl}_{3}\right): \delta 154.71,132.10,131.89,124.54,121.63,110.54,55.63,41.15$. 
For 4d. Yield, $106 \mathrm{mg}, 98 \%$. ESI-MS: $m / z=218.94[\mathrm{M}+\mathrm{H}]^{+} .{ }^{1} \mathrm{H}$ NMR $\left(300 \mathrm{MHz}, \mathrm{CDCl}_{3}\right) \delta: 7.93(\mathrm{~d}, J=$ $7.8 \mathrm{~Hz}, 1 \mathrm{H}), 7.59-7.53(\mathrm{~m}, 2 \mathrm{H}), 7.35(\mathrm{~m}, 1 \mathrm{H}), 2.80(\mathrm{~s}, 3 \mathrm{H}) .{ }^{13} \mathrm{C} \mathrm{NMR}\left(101 \mathrm{MHz}, \mathrm{CDCl}_{3}\right): \delta 145.43$, $133.04,132.39,128.86,125.75,118.49,42.04$.

For 4e. Yield, $100 \mathrm{mg}, 93 \%$. ESI-MS: $m / z=217.07[\mathrm{M}+\mathrm{H}]^{+} .{ }^{1} \mathrm{H}$ NMR $\left(400 \mathrm{MHz}, \mathrm{CDCl}_{3}\right) \delta 7.44-7.32$ (m, 5H), 7.25-7.17 (m, 3H), $6.95(\mathrm{~m}, 2 \mathrm{H}), 4.04(\mathrm{~d}, J=12.6 \mathrm{~Hz}, 1 \mathrm{H}), 3.96(\mathrm{~d}, J=12.6 \mathrm{~Hz}, 1 \mathrm{H}) .{ }^{13} \mathrm{C}$ NMR (101 MHz, $\left.\mathrm{CDCl}_{3}\right): \delta 142.66,131.05,130.26,130.26,129.05,128.74,128.70,128.32,128.30$, 128.04, 124.30, 124.24, 63.39.

For 4f. Yield, $87 \mathrm{mg}, 95 \%$. ESI-MS: $m / z=183.05[\mathrm{M}-\mathrm{H}]^{-} .{ }^{1} \mathrm{H}$ NMR $\left(400 \mathrm{MHz}, \mathrm{CDCl}_{3}\right): \delta 10.65(\mathrm{~s}, 1 \mathrm{H})$, 7.39 (t, $J=7.5 \mathrm{~Hz}, 1 \mathrm{H}), 7.02(\mathrm{~d}, J=7.6 \mathrm{~Hz}, 1 \mathrm{H}), 6.93(\mathrm{dd}, J=15.4,8.0 \mathrm{~Hz}, 2 \mathrm{H}), 3.30-3.15(\mathrm{~m}, 1 \mathrm{H})$, $1.39(\mathrm{~d}, J=6.8 \mathrm{~Hz}, 3 \mathrm{H}), 1.29(\mathrm{~d}, J=6.8 \mathrm{~Hz}, 3 \mathrm{H})$.

For 4g. Yield, $111 \mathrm{mg}, 96 \%$. ESI-MS: $m / z=231.06[\mathrm{M}-\mathrm{H}]^{-} .{ }^{1} \mathrm{H}$ NMR $\left(400 \mathrm{MHz}, \mathrm{CDCl}_{3}\right): \delta 10.18(\mathrm{~s}$, 1H), 7.72-7.64 (m, 2H), 7.52-7.44 (m, 3H), $7.14(\mathrm{~d}, J=7.9 \mathrm{~Hz}, 1 \mathrm{H}), 6.77-6.69(\mathrm{~m}, 2 \mathrm{H}), 2.28(\mathrm{~s}, 3 \mathrm{H}) .{ }^{13} \mathrm{C}$ NMR (101 MHz, $\left.\mathrm{CDCl}_{3}\right): \delta 159.33,144.32,144.04,131.41,129.58,129.58,126.04,124.85,124.85$, $121.05,121.05,119.98,21.63$.

3 General Procedure for Release of Chiral Sulfoxide and Recovery of the Chiral Ir(III) Complex. A suspension of sulfoxide complexes $\Lambda$-[Ir(dfppy $\left.)_{2}\{(S)-\mathbf{4 f}\}\right](0.03 \mathrm{mmol})$ in acetonitrile $(5 \mathrm{~mL})$ was stirred and degassed by $\mathrm{N}_{2}$ at $55^{\circ} \mathrm{C}$ for $5 \mathrm{~min}$, and then TFA $(27 \mu \mathrm{L}, 0.36 \mathrm{mmol})$ was added to the solution. The solution was stirred at $55^{\circ} \mathrm{C}$ for another $1 \mathrm{~h}$. After that, the reaction solution was cooled to room temperature, and the solvent was concentrated to dryness. The residue material was dissolved in $\mathrm{CHCl}_{3}(0.5 \mathrm{~mL})$, and then hexane $(15 \mathrm{~mL})$ was added to the above solution to produce precipitate. The precipitate was filtrated, washed with $\mathrm{CHCl}_{3}$-hexane (1:30) and then with a $\mathrm{KPF}_{6}$ solution to afford $\Lambda$-[Ir(dfppy) $\left.)_{2}(\mathrm{MeCN})_{2}\right]\left(\mathrm{PF}_{6}\right)$. Yield, 89\%. ${ }^{1} \mathrm{H}$ NMR $\left(400 \mathrm{MHz}, \mathrm{CD}_{3} \mathrm{CN}\right) \delta 9.08(\mathrm{~d}, J=5.6 \mathrm{~Hz}, 2 \mathrm{H}), 8.35$ $(\mathrm{d}, J=8.4 \mathrm{~Hz}, 2 \mathrm{H}), 8.11(\mathrm{t}, J=7.8 \mathrm{~Hz}, 2 \mathrm{H}), 7.51(\mathrm{t}, J=6.6 \mathrm{~Hz}, 2 \mathrm{H}), 6.56(\mathrm{~d}, J=11.2 \mathrm{~Hz}, 2 \mathrm{H}), 5.53(\mathrm{~d}$, $J=8.8 \mathrm{~Hz}, 2 \mathrm{H}), 2.14(\mathrm{~s}, 6 \mathrm{H})$. The filtrate was concentrated to dryness and subjected to purification on a silica gel chromatography with DCM as an eluent to afford colorless solid as $(R)-\mathbf{4 f}$. When $\Delta$-Ir- $R-\mathbf{4 f}$, $\Delta$-Ir-R-4g or $\Lambda$-Ir-S-4g was used instead of $\Lambda$-Ir-S-4f, the corresponding sulfoxides were obtained. 


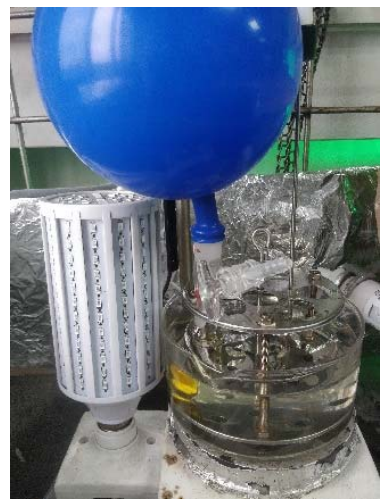

Figure S1. A photograph of the photoreaction setup.

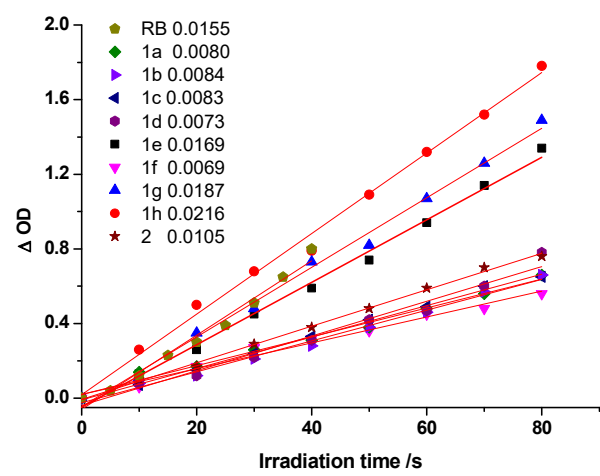

Figure S2. Plot of change in absorbance of DPBF at $414 \mathrm{~nm}$ vs irradiation time in the presence of $\operatorname{Ir}(\mathrm{III})$ complexes against Rose Bengal (RB) as the standard in $\mathrm{MeOH}$ solution. 

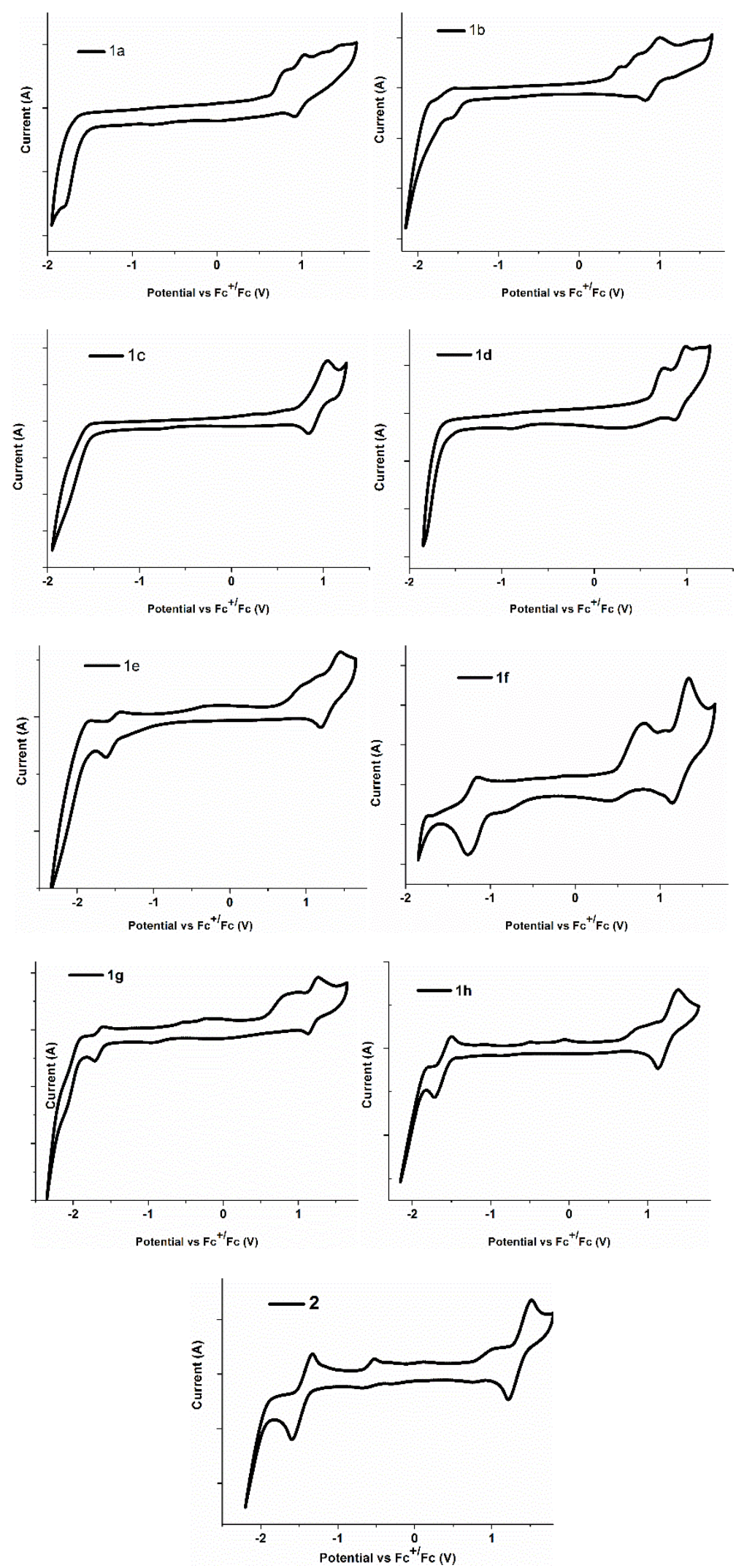

Figure S3. Cyclic voltammograms of $\mathrm{Ir}(\mathrm{III})$ complexes in degassed $\mathrm{CH}_{2} \mathrm{Cl}_{2}$ solution at a scan rate of 200 $\mathrm{mV} / \mathrm{s}$. 


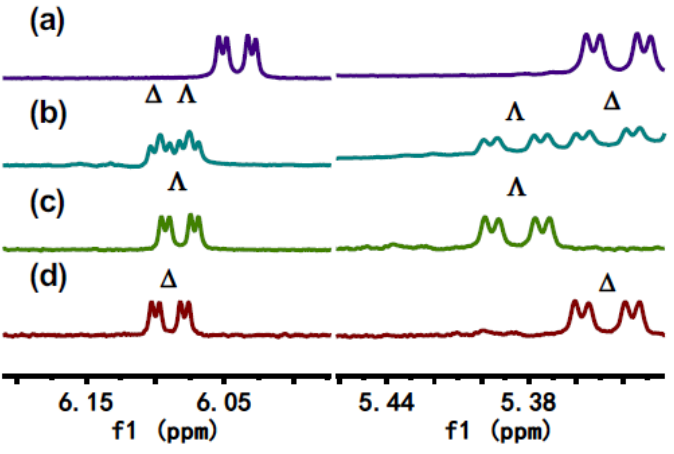

Figure S4. Excerpts of ${ }^{1} \mathrm{H}$ NMR spectra in $\mathrm{CDCl}_{3}$ : (a) rac-4g, (b) rac-4g with 50 equiv of S-binol, (c) $\Lambda$-Ir-S-4g with 50 equiv of S-binol, and (d) $\Delta$-Ir-R-4g with 50 equiv of S-binol.
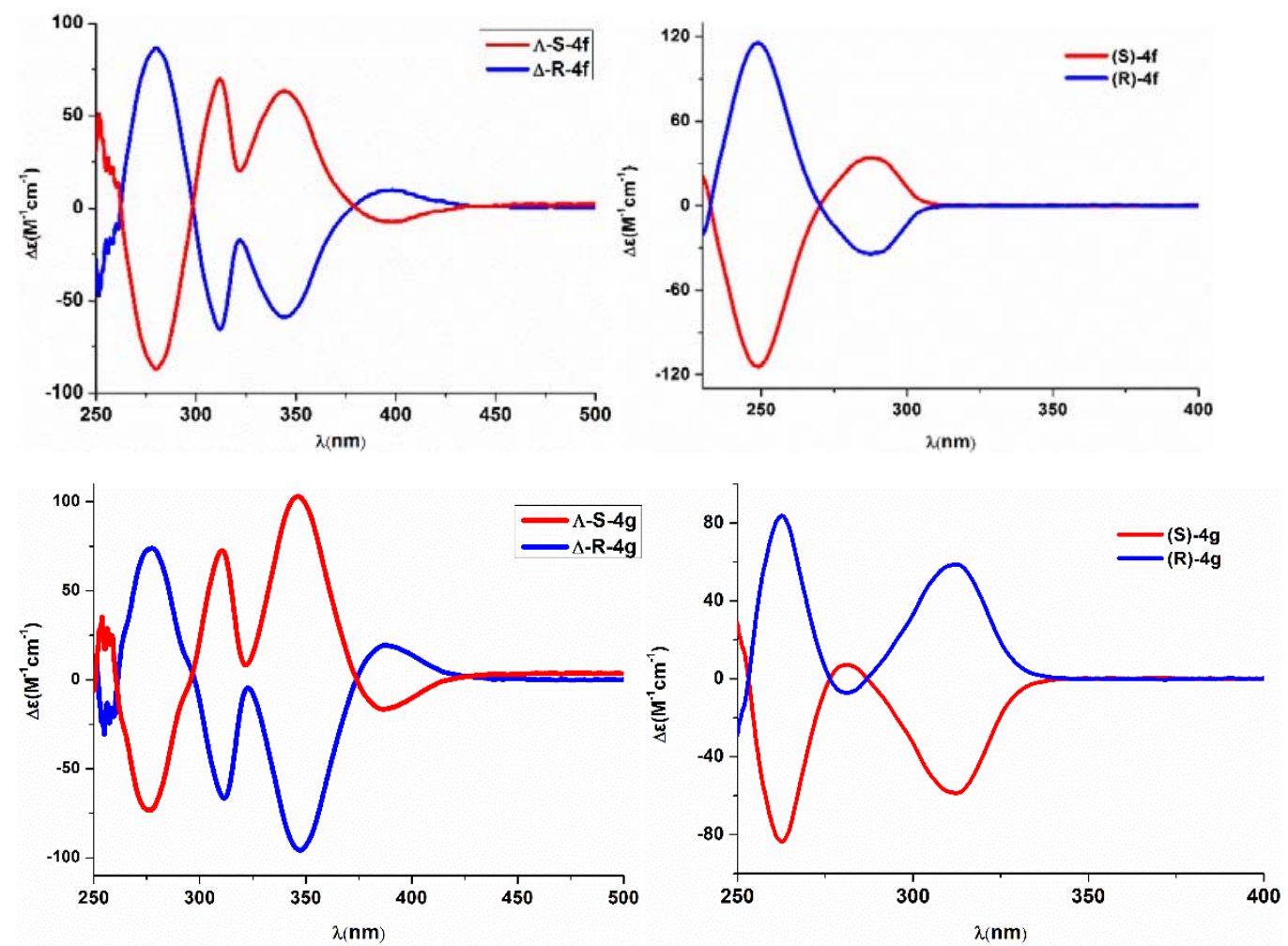

Figure S5. CD spectra of enantiomeric sulfoxide complexes and sulfoxides in $\mathrm{CH}_{2} \mathrm{Cl}_{2}$ solution $\left(1.0 \times 10^{-5} \mathrm{M}\right)$.
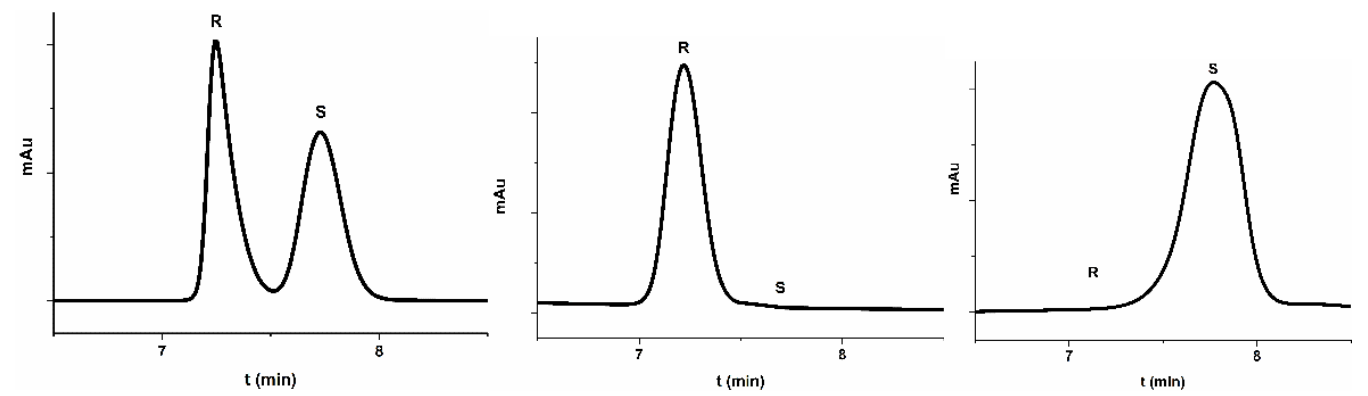

Figure S6. HPLC traces of $\mathbf{4 f}$ (left), $(R)-\mathbf{4 f}$ with $98.9 \%$ ee value (middle), and (S)-4f with $99.2 \%$ ee value (right). HPLC conditions: Daicel Chiralpak AD-H column, $250 \mathrm{~mm} \times 4.6 \mathrm{~mm}$, flow rate $=1 \mathrm{~mL} / \mathrm{min}$, column temperature $=30^{\circ} \mathrm{C}, 300 \mathrm{~nm}$, hexane $/(\mathrm{EtOH}: \mathrm{MeOH}) / \mathrm{TFA}=90 / 10(3: 1) / 0.1$ as an eluent. 

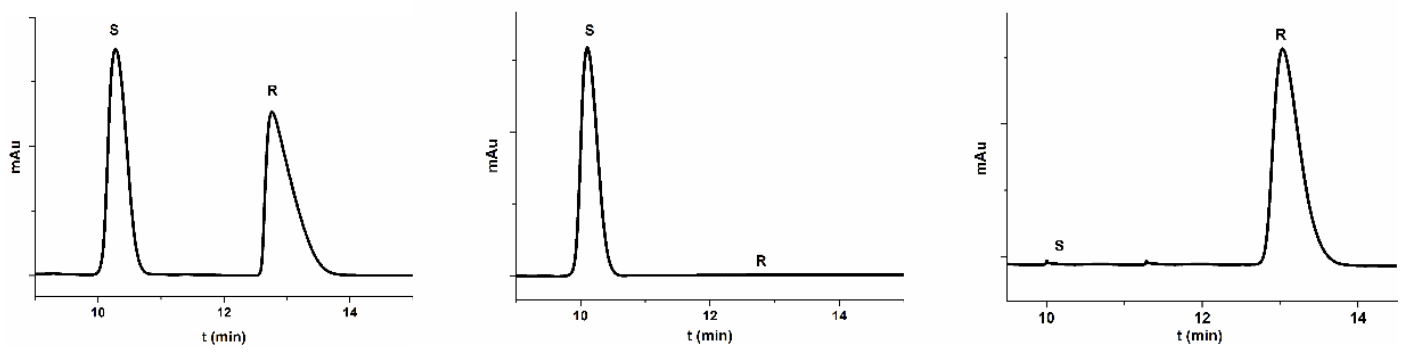

Figure S7. HPLC traces of $\mathbf{4 g}$ (left), $(S)-\mathbf{4 g}$ with $99.8 \%$ ee value (middle), and $(R)-\mathbf{4 g}$ with $98.0 \%$ ee value (right). HPLC conditions: Daicel Chiralpak AD-H column, $250 \mathrm{~mm} \times 4.6 \mathrm{~mm}$, flow rate $=1 \mathrm{~mL} / \mathrm{min}$, column temperature $=30^{\circ} \mathrm{C}, 300 \mathrm{~nm}$, hexane $/(\mathrm{EtOH}: \mathrm{MeOH}) / \mathrm{TFA}=90 / 10(3: 1) / 0.1$ as an eluent.

Table S1. Crystallographic Data for $1 \mathrm{~b} \cdot \mathrm{CH}_{2} \mathrm{Cl}_{2}, \mathbf{1 c} \cdot \mathrm{CH}_{3} \mathrm{OH}$, 1e, and $1 \mathrm{~h}$

\begin{tabular}{|c|c|c|c|c|}
\hline Complex & $1 \mathrm{~b} \cdot \mathrm{CH}_{2} \mathrm{Cl}_{2}$ & $1 \mathrm{c} \cdot \mathrm{CH}_{3} \mathrm{OH}$ & $1 \mathrm{e}$ & $1 \mathrm{~h}$ \\
\hline $\begin{array}{l}\text { Molecular } \\
\text { formula }\end{array}$ & $\mathrm{C}_{43} \mathrm{~F}_{6} \mathrm{IrO}_{2} \mathrm{PN}_{4} \mathrm{Cl}_{2} \mathrm{H}_{36}$ & $\mathrm{C}_{45} \mathrm{H}_{44} \mathrm{~F}_{6} \mathrm{IrN}_{6} \mathrm{OP}$ & $\mathrm{C}_{40} \mathrm{H}_{28} \mathrm{~F}_{6} \mathrm{IrN}_{4} \mathrm{P}$ & $\mathrm{C}_{42} \mathrm{H}_{32} \mathrm{~F}_{6} \mathrm{IrN}_{4} \mathrm{O}_{2} \mathrm{P}$ \\
\hline$M_{\mathrm{r}}$ & 1048.83 & 1022.03 & 901.83 & 961.89 \\
\hline $\begin{array}{l}\text { Crystal } \\
\text { system }\end{array}$ & monoclinic & monoclinic & monoclinic & orthorhombic \\
\hline Space group & $P 2_{1} / c$ & $P 2 / c$ & $P 2_{1} / c$ & $I b c a$ \\
\hline Temperature & 298 & 298 & 298 & 298 \\
\hline$a / \AA ̊$ & $14.3154(8)$ & $15.7815(3)$ & $13.0845(6)$ & $17.9795(15)$ \\
\hline$b / \AA$ & $18.1516(11)$ & $11.5709(2)$ & $14.1519(7)$ & $18.1404(16)$ \\
\hline$c / \AA ̊$ & $18.227(1)$ & $24.1607(5)$ & $18.5764(8)$ & 26.193(3) \\
\hline$\beta /$ & 119.297(4) & $107.738(2)$ & $91.126(4)$ & 90.00 \\
\hline$V / \AA^{3}$ & $4130.4(4)$ & $4202.15(14)$ & $3439.1(3)$ & $8543.0(14)$ \\
\hline$Z$ & 4 & 4 & 4 & 8 \\
\hline$D_{c}\left(\mathrm{~g} \mathrm{~cm}^{-3}\right)$ & 1.687 & 1.615 & 1.742 & 1.496 \\
\hline$\mu\left(\mathrm{mm}^{-1}\right)$ & 3.470 & 7.109 & 8.557 & 3.227 \\
\hline $\begin{array}{l}\text { no. data of } \\
\text { collected }\end{array}$ & 22676 & 22179 & 15444 & 36788 \\
\hline $\begin{array}{l}\text { no. observed } \\
\text { reflections }\end{array}$ & 9181 & 6724 & 5795 & 4895 \\
\hline${ }^{a} R_{1}[I>2 \sigma(\mathrm{I})]$ & 0.0616 & 0.0319 & 0.0857 & 0.0439 \\
\hline${ }^{b} \omega R_{2}$ (all data) & 0.1969 & 0.0835 & 0.3095 & 0.1514 \\
\hline GOF & 1.006 & 1.033 & 1.179 & 1.032 \\
\hline $\mathrm{CCDC}$ & 1892575 & 1892576 & 1892577 & 1892578 \\
\hline${ }^{\mathrm{a}} R_{1}=\Sigma|| F_{0}|-| F_{\mathrm{c}}||$ & $\Sigma\left|F_{0}\right| \cdot{ }^{b} \omega R_{2}=\left[\Sigma \omega\left(F_{0}{ }^{2}\right.\right.$ & $\left.\left.F_{\mathrm{c}}\right)^{2} / \Sigma \omega\left(F_{0}^{2}\right)^{2}\right]^{1 / 2}$ & & \\
\hline
\end{tabular}

